PROCEEDINGS OF THE

AMERICAN MATHEMATICAL SOCIETY

Volume 132, Number 1, Pages 165-174

S 0002-9939(03)06979-X

Article electronically published on May 7, 2003

\title{
SEQUENTIAL AND CONTINUUM BIFURCATIONS IN DEGENERATE ELLIPTIC EQUATIONS
}

\author{
R. E. BEARDMORE AND R. LAISTER \\ (Communicated by Carmen C. Chicone)
}

\begin{abstract}
We examine the bifurcations to positive and sign-changing solutions of degenerate elliptic equations. In the problems we study, which do not represent Fredholm operators, we show that there is a critical parameter value at which an infinity of bifurcations occur from the trivial solution. Moreover, a bifurcation occurs at each point in some unbounded interval in parameter space. We apply our results to non-monotone eigenvalue problems, degenerate semi-linear elliptic equations, boundary value differential-algebraic equations and fully non-linear elliptic equations.
\end{abstract}

\section{INTRODUCTION}

In this paper we consider the non-linear, degenerate eigenvalue problem

$$
\begin{array}{cl}
L g(u)=\lambda u, & x \in \Omega:=(0,1), \\
u=0, & x \in \partial \Omega,
\end{array}
$$

where $L u:=-\left(a(x) u_{x}\right)_{x}+b(x) u$ and the coefficients $a, b \in C^{1}(\bar{\Omega})$ satisfy $a>0$ and $b \geq 0$ on $\bar{\Omega}$. Consequently $L$ is uniformly elliptic, but the non-linear function $g \in C^{1}(\mathbb{R})$ is assumed to degenerate at zero with $g(0)=g^{\prime}(0)=0$.

Let us define $\gamma(u)=g(u) / u$ with $\gamma(0)=0$ and begin with the statement of our assumptions on $g$ :

G1. $g$ is an odd, strictly increasing function on $\mathbb{R}$,

G2. $u>0$ implies $\gamma^{\prime}(u)>0$,

G3. $\gamma(u) \rightarrow \infty$ as $|u| \rightarrow \infty$.

These are all satisfied if, for instance, $g(u)=u|u|^{m}$, where $m>0$.

Definition 1.1. Let $X, Y$ be Banach spaces, $F: X \times \mathbb{R} \rightarrow Y$ be continuous and satisfy $F(0, \lambda)=0$ for all $\lambda \in \mathbb{R}$. Let $\Sigma \subset X \times \mathbb{R}$ denote the set of all non-trivial $(u \neq 0)$ solutions of $F(u, \lambda)=0$. We say that $\lambda_{0}$ is a sequential bifurcation point from the trivial solution for $F(u, \lambda)=0$ if there is a sequence $\left(u_{n}, \lambda_{n}\right) \in \Sigma$ such that $\left(u_{n}, \lambda_{n}\right) \rightarrow\left(0, \lambda_{0}\right)$ in $X \times \mathbb{R}$ as $n \rightarrow \infty$. If such a sequence $\left(u_{n}, \lambda_{n}\right)$ lies in some connected set $\mathcal{C} \subset \Sigma$, then $\lambda_{0}$ is said to be a continuum bifurcation point.

Received by the editors May 13, 2002 and, in revised form, August 21, 2002.

1991 Mathematics Subject Classification. Primary 34A09, 34B60, 35B32, 35J60, 35J70.

Key words and phrases. Degenerate elliptic equations, sequential and continuum bifurcations, differential-algebraic equations, degenerate diffusion. 
We prove the following for (11)-(2). To each $\lambda>0$ there is a sequence $u_{n}(\lambda) \in$ $C^{0}(\bar{\Omega})$ of solutions of (11)-(2) such that (i) the number of zeros of $u_{n}(\lambda)$ in $\Omega$ is $n$, (ii) $u_{n}(\lambda) \rightarrow 0$ in $C^{0}(\bar{\Omega})$ as $n \rightarrow \infty$, (iii) $u_{n}(\lambda) \rightarrow 0$ in $C^{0}(\bar{\Omega})$ as $\lambda \rightarrow 0$, (iv) every $\lambda>0$ is a sequential bifurcation point but not a continuum bifurcation point and (v) $\lambda=0$ is a continuum bifurcation point.

We remark that the theory in 11 could be used to obtain local versions of some of the results proved here. However, our results are complementary to [1] in that they are global and impose no conditions on the growth of $g^{-1}$ near zero. Furthermore, we establish the existence of an unbounded interval of sequential bifurcation points. For the special case $g(u)=u|u|^{m}$, we note that a global branch of positive solutions was shown to exist in [2] in a study of flows in porous media.

The remainder of the paper is structured as follows. Section 2 introduces some notation and preliminary results. The main results of the paper appear in Section 3. Finally, in Section 4 we apply our results to non-monotone degenerate eigenvalue problems, degenerate semi-linear elliptic equations, boundary value differentialalgebraic equations and fully non-linear elliptic equations.

\section{Preliminaries}

Throughout we write $\bar{U}$ for the closure of $U$ in a given metric space. We denote by $C^{k}(\bar{\Omega})$ the space of $k$-times differentiable functions on $\bar{\Omega}$, henceforth written simply as $C^{k}$ when there is no ambiguity. We note here that the imbedding $C^{k} \hookrightarrow C^{r}$ is compact if $k>r$. For any $u \in C^{0}$ with finitely many zeros we shall denote the number of zeros of $u$ in $\Omega$ by $\zeta(u)$.

It is well known that $L: C^{2} \rightarrow C^{0}$ together with the Dirichlet boundary condition (2) has positive, simple eigenvalues, henceforth denoted by $\mu_{j}$ for $j \in \mathbb{N}_{0}:=\mathbb{N} \cup$ $\{0\}$, where the principal eigenvalue $\mu_{0}$ has an associated positive eigenfunction $\phi_{0}$. Furthermore, $L$ has a continuous inverse $K: C^{0} \rightarrow C^{2}$ which induces a compact linear map $K: C^{0} \rightarrow C^{0}$.

The problem of finding continuous solutions of (1)-(2) with $g(u(\cdot)) \in C^{2}$ is therefore equivalent to

$$
F(u, \lambda):=g(u)-\lambda K u=0, \quad u \in C^{0},
$$

where $g: C^{0} \rightarrow C^{0}$ is the $C^{1}$ Nemytskii operator for $g$ defined by $(g(u))(x)=$ $g(u(x))$. Our approach to solving (3) will be based on the regularized problem

$$
F(u, \lambda ; \varepsilon):=g(u)+(\varepsilon I-\lambda K) u=0, \quad \varepsilon \geq 0 .
$$

We define some solution sets. Throughout $E:=C^{0} \times \mathbb{R}$ is endowed with the norm $\|(u, \lambda)\|_{E}=\|u\|+|\lambda|$, where $\|\cdot\|$ denotes the sup-norm on $C^{0}$. The symbol $\langle\cdot, \cdot\rangle$ denotes the usual $L^{2}$ inner product. For $\varepsilon \geq 0, \Sigma(\varepsilon) \subset E$ will denote the set of non-trivial solutions $(u, \lambda)$ of $F(u, \lambda ; \varepsilon)=0$ in $E$. For $j \in \mathbb{N}_{0}$ we write $\Sigma_{j}(\varepsilon)$ for the subset of $\Sigma(\varepsilon)$ consisting of functions with $j$ zeros in $\Omega$. By $\Sigma_{j}^{+}(\varepsilon)\left(\Sigma_{j}^{-}(\varepsilon)\right)$ we denote the subset of $\Sigma_{j}(\varepsilon)$ of functions $u$ such that $g(u)_{x}(0)>0\left(g(u)_{x}(0)<0\right)$. For notational convenience we will simply write $\Sigma$ instead of $\Sigma(0)$ and $\Sigma_{j}^{ \pm}$for $\Sigma_{j}^{ \pm}(0)$. We note here that since $g$ is odd, $(u, \lambda) \in \Sigma(\varepsilon)$ if and only if $(-u, \lambda) \in \Sigma(\varepsilon)$. Consequently $\Sigma_{j}^{-}(\varepsilon)=-\Sigma_{j}^{+}(\varepsilon)$.

Remark 1. The map $F: C^{0} \times \mathbb{R} \rightarrow C^{0}$ is $C^{1}$ and has partial Fréchet derivative $d_{u} F(u, \lambda)[h]=g^{\prime}(u) h-\lambda K h$ which is not a Fredholm mapping at $u=0$ since $g^{\prime}(0)=0$. Consequently, one cannot use reduction methods based on the implicit 
function theorem to study bifurcations of (3) from the trivial solution. See also [3, 4, 15. Moreover, $d_{u} F(0, \lambda)-\lambda K$ which, for $\lambda \neq 0$, has point spectrum and zero in the essential spectrum, but when $\lambda=0$, the spectrum consists only of zero.

Lemma 2.1. Fix $\varepsilon \geq 0$. If $(u, \lambda) \in \Sigma(\varepsilon)$, then $\lambda>0$; that is, $\Sigma(\varepsilon) \subset C^{0} \times(0, \infty)$.

Proof. Multiplying the relation $F(u, \lambda ; \varepsilon)=0$ by $u$ and integrating over $\Omega$ gives, after setting $v=K u$,

$$
\int_{\Omega} \varepsilon u^{2}+u g(u) d x=\lambda \int_{\Omega} u K u d x=\lambda \int_{\Omega} v L v d x .
$$

Noting that $u g(u) \geq 0$ and $\langle v, L v\rangle \geq 0$, the result follows.

Lemma 2.2. For $\varepsilon \in[0,1]$ the following a priori bound applies: to each $\ell>0$ there is an $M(\ell)>0$, independent of $\varepsilon$, such that if $\lambda \in[0, \ell]$, then $\|u\| \leq M(\ell)$ whenever $(u, \lambda) \in \Sigma(\varepsilon)$.

Proof. Suppose that $\varepsilon u+g(u)=\lambda K u$, where $0 \leq \varepsilon \leq 1,0 \leq \lambda \leq \ell$ and let $x_{0} \in \Omega$ satisfy $\|u\|=\left|u\left(x_{0}\right)\right|$. Then

$$
|| g\left(u\left(x_{0}\right)\right)|-|-\varepsilon u\left(x_{0}\right)|| \leq\left|g\left(u\left(x_{0}\right)\right)+\varepsilon u\left(x_{0}\right)\right| \leq \lambda\|K\|\left|u\left(x_{0}\right)\right|,
$$

where $\|K\|$ denotes the operator norm of $K \in B L\left(C^{0}\right)$. We therefore obtain $\gamma(\|u\|) \leq \lambda\|K\|+\varepsilon \leq \ell\|K\|+1$. Noting that $\gamma \mid:[0, \infty) \rightarrow[0, \infty)$ is surjective (by G3) and non-decreasing (by G2), the result follows on defining $M(\ell)$ to be any positive solution of $\gamma(M)=\ell\|K\|+1$.

Since $\varepsilon+g^{\prime}(u) \geq \varepsilon>0$ for all $u \in \mathbb{R}$ and $\varepsilon>0$, the algebraic equation $\varepsilon u+g(u)=$ $v$ has a unique solution $u=G(v ; \varepsilon)$, where $G(\cdot ; \varepsilon) \in C^{1}(\mathbb{R})$. When $\varepsilon=0$ we simply have $G(v ; 0)=g^{-1}(v)$, which is continuous. Moreover, $G: \mathbb{R} \times[0, \infty) \rightarrow \mathbb{R}$ is continuous. We shall use this notation throughout and in the following theorem, which is a consequence of global bifurcation theory.

Theorem 2.3. For each $\varepsilon>0$ and $j \in \mathbb{N}_{0}$, there are open, connected and unbounded sets $C_{j}^{ \pm}(\varepsilon) \subset \Sigma_{j}^{ \pm}(\varepsilon)$ such that $\left(0, \varepsilon \mu_{j}\right) \in \overline{C_{j}^{ \pm}(\varepsilon)}$. Furthermore, for every $\lambda>\varepsilon \mu_{j}$ there exist $\left( \pm u_{j, \varepsilon}, \lambda\right) \in C_{j}^{ \pm}(\varepsilon)$, so that $\left(\varepsilon \mu_{j}, \infty\right) \subset \Pi\left(C_{j}^{ \pm}(\varepsilon)\right)$, where $\Pi: E \rightarrow \mathbb{R}$ is the natural projection.

Proof. For each fixed $\varepsilon>0$, apply global bifurcation results [13] to $v=\lambda K G(v ; \varepsilon)$ and use the nodal properties of solutions to regular elliptic equations to demonstrate the existence of disjoint, unbounded continua $C_{j}^{ \pm}(\varepsilon)$ with the stated properties. The existence of $\left( \pm u_{j, \varepsilon}, \lambda\right)$ for $\lambda>\varepsilon \mu_{j}$ follows from the unboundedness of $C_{j}^{ \pm}(\varepsilon)$ in $E$, Lemma 2.1 and Lemma 2.2 .

If $u$ is a non-trivial solution of (4) with $\varepsilon>0$, then the zeros of the function $\varepsilon u+g(u)$ are transverse. The following result shows that transversality persists when $\varepsilon=0$.

Theorem 2.4 (see [9, Theorem 2.2]). Suppose that $f \in C^{0}(\mathbb{R})$ is strictly increasing and $f(0)=0$. If $u \in C^{2}(\bar{\Omega})$ is a solution of the initial value problem $L u=f(u)$ on $\bar{\Omega}$ with $u(\alpha)=u_{x}(\alpha)=0$ for some $\alpha \in \bar{\Omega}$, then $u \equiv 0$ on $\bar{\Omega}$. Furthermore, $u$ has a finite number of zeros in $\bar{\Omega}$.

Corollary 2.5. If $(u, \lambda) \in \Sigma$, then $\zeta(u)=\zeta(g(u))<\infty$ and all zeros of $g(u)$ in $\bar{\Omega}$ are transverse. In particular, $\Sigma=\bigcup_{j=0}^{\infty}\left(\Sigma_{j}^{+} \cup \Sigma_{j}^{-}\right)$. 
Proof. If $(u, \lambda) \in \Sigma$ and $v:=g(u)$, then $L v=\lambda g^{-1}(v)$. The result follows from Lemma 2.1] and Theorem 2.4 with $f(v)=\lambda g^{-1}(v)$.

\section{The MAIN RESUlts}

In this section we prove the main results on the existence of non-trivial solutions of (3) and the nature of bifurcation points.

3.1. Existence of non-trivial solutions. We begin with an existence and uniqueness result for elliptic equations.

Lemma 3.1. Suppose $A u:=-\left(\alpha(x) u_{x}\right)_{x}+\beta(x) u$, where $\alpha$ and $\beta$ satisfy the same assumptions as $a$ and $b$. Let $\lambda>0$ and $\varepsilon \geq 0$ be fixed. If there exists a positive subsolution $\psi$ of the elliptic problem

$$
A v=\lambda G(v ; \varepsilon), \quad v(0)=v(1)=0,
$$

then there exists a unique non-trivial, non-negative solution $v$ of (5). Moreover, $v \geq \psi$.

Proof. By assumption G3, $\lim _{v \rightarrow \infty} G(v ; \varepsilon) / v=0$ for fixed $\varepsilon \geq 0$. In particular this implies that $\lim \sup _{v \rightarrow \infty} \lambda G(v ; \varepsilon) / v<\kappa_{0}$, where $\kappa_{0}$ denotes the principal eigenvalue of $A$. It is well known [6, 11] that non-negative solutions of the associated parabolic problem

$$
v_{t}=-A v+\lambda G(v ; \varepsilon), \quad v(0, t)=v(1, t)=0
$$

(with continuous initial condition $v(x, 0)=v_{0}(x)$ ) have non-empty omega-limit sets $\omega\left(v_{0}\right)$ contained in the equilibrium set, comprising of solutions of (5). In particular, since $\psi$ is also a subsolution of (6), there exists a solution $v$ of (5) such that $v \geq \psi$. It therefore remains only to establish the uniqueness of $v$.

Suppose $w$ is any non-trivial, non-negative solution of (5). By G1 and the maximum principle, $w>0$ in $\Omega$. Now, $\int_{0}^{1} v A w-w A v d x=0$ so that

$$
\lambda \int_{0}^{1} v G(w ; \varepsilon)-w G(v ; \varepsilon) d x=\int_{0}^{1} \lambda v w\left(\frac{G(w ; \varepsilon)}{w}-\frac{G(v ; \varepsilon)}{v}\right) d x=0 .
$$

By G2, $s \mapsto G(s ; \varepsilon) / s$ is decreasing for all $s>0$. Hence if $v$ and $w$ are ordered in $C^{0}$, then $v=w$ and $v$ is unique. If $v$ and $w$ are not ordered in $C^{0}$, then, for any $v_{0} \geq \max \{v, w\}, \omega\left(v_{0}\right)$ must contain a solution $z$ of (5) such that $z \geq \max \{v, w\}$, whence $z \neq v$ and $z \neq w$. Hence $z$ and $v$ are ordered in $C^{0}$ and the above argument (with $w$ replaced by $z$ ) yields $z=v$, a contradiction.

The following result is crucial, showing that non-trivial $j$-zero solutions of the regularized problem (44) cannot accumulate on the trivial branch as $\varepsilon \rightarrow 0$, except possibly at the origin.

Proposition 3.2. Let $j \in \mathbb{N}_{0}$ be fixed and $0 \leq \varepsilon_{n} \rightarrow 0$ as $n \rightarrow \infty$. If $\left(u_{n}, \lambda_{n}\right) \in$ $\Sigma_{j}^{+}\left(\varepsilon_{n}\right)$ satisfies $\left(u_{n}, \lambda_{n}\right) \rightarrow(0, \lambda)$ in $E$ as $n \rightarrow \infty$, then $\lambda=0$. An analogous result holds for $\Sigma_{j}^{-}\left(\varepsilon_{n}\right)$.

Proof. Necessarily $\lambda \geq 0$ by Lemma 2.1 so suppose that $\lambda>0$. We first consider the case $j=0$ (positive solutions). Fix $\lambda_{*} \in(0, \lambda)$ and choose $n_{0}$ such that $\varepsilon_{n}<\min \left\{\mu_{0},\left(\lambda_{*} / \mu_{0}\right)\right\}$ and $\lambda_{n}>\lambda_{*}$ for all $n>n_{0}$. By the degeneracy of $g$ there is a $U>0$ (independent of $n$ ) such that $g(u)+\varepsilon_{n} u \leq\left(\lambda_{*} / \mu_{0}\right) u$ for all $u \in[0, U]$ and $n>n_{0}$. Hence there is a $V>0$ (independent of $\left.n\right)$ such that $G\left(v ; \varepsilon_{n}\right) \geq\left(\mu_{0} / \lambda_{*}\right) v$ 
for all $v \in[0, V]$ and $n>n_{0}$. Let us normalise the principal eigenfunction of $L, \phi_{0}$, so that $\left\|\phi_{0}\right\|=V$. Since $G\left(\phi_{0}, \varepsilon_{n}\right) \geq\left(\mu_{0} / \lambda_{*}\right) \phi_{0}$ it follows that

$$
-L \phi_{0}+\lambda_{n} G\left(\phi_{0} ; \varepsilon_{n}\right) \geq-L \phi_{0}+\lambda_{n}\left(\mu_{0} / \lambda_{*}\right) \phi_{0} \geq 0
$$

and so $\phi_{0}$ is a subsolution of

$$
L v=\lambda_{n} G\left(v ; \varepsilon_{n}\right), \quad v(0)=v(1)=0 .
$$

Hence by Lemma 3.1 there exists a unique positive solution $w_{n}$ of (7) and $w_{n} \geq \phi_{0}$. Now, $v_{n}:=\varepsilon_{n} u_{n}+g\left(u_{n}\right)$ is also a positive solution of (17) and so by uniqueness $v_{n}=w_{n}$. But since $v_{n}=\lambda_{n} K u_{n}$ and $u_{n} \rightarrow 0$ in $C^{0}$ as $n \rightarrow \infty$, it follows that $v_{n} \rightarrow 0$ in $C^{2}$. In particular, by Hopf's boundary point lemma [12] applied to $\phi_{0}$, there exists an $n_{1}>n_{0}$ such that $v_{n}<\phi_{0}$ in $\Omega$ for all $n>n_{1}$, a contradiction. This proves the result for $j=0$. The result for $\Sigma_{j}^{-}\left(\varepsilon_{n}\right)$ is a trivial consequence of the symmetry of $g$.

Now suppose that $j \geq 1$. If $\xi_{n}^{i}(i=0, \ldots, j+1)$ denote the zeros of $u_{n}$ in $\bar{\Omega}$ in increasing order, let $\delta_{n}^{i}=\xi_{n}^{i+1}-\xi_{n}^{i}(i=0, \ldots, j)$. Then $v_{n}:=\varepsilon_{n} u_{n}+g\left(u_{n}\right)$ (suitably restricted) is a constant sign solution of

$$
L v=\lambda_{n} G\left(v ; \varepsilon_{n}\right), \quad v\left(\xi_{n}^{i}\right)=v\left(\xi_{n}^{i+1}\right)=0 .
$$

Since $\sum_{i=0}^{j} \delta_{n}^{i} \equiv 1$, we can assume for some $i$ that $\delta_{n}^{i}$ (=: $\left.\delta_{n}\right)$ remains uniformly bounded away from zero. Passing to a subsequence if necessary we may assume that $\delta_{n} \rightarrow \delta_{\infty} \in(0,1]$ as $n \rightarrow \infty$. Now rescale the spatial variable $x$ in (8) according to $x \mapsto\left(x-\xi_{n}^{i+1}\right) / \delta_{n}$ and, without loss of generality by the symmetry of $g$, we obtain a sequence $v_{n}$ of positive solutions of

$$
L_{n} v \lambda_{n} G\left(v ; \varepsilon_{n}\right), \quad v(0)=v(1)=0,
$$

with $v_{n} \rightarrow 0$ in $C^{2}$, where $L_{n} v:=-\delta_{n}^{-2}\left(a(x) v_{x}\right)_{x}+b(x) v$. If we denote by $\left\{\mu_{0}^{n}, \phi_{0}^{n}\right\}$ the principal eigenpair of the operator $L_{n}$, then spectral perturbation results for simple eigenvalues [7] show that $\mu_{0}^{n} \rightarrow \mu_{0}^{\infty}$, the principal eigenvalue of $L_{\infty}$, and $\phi_{0}^{n} \rightarrow \phi_{0}^{\infty}$ in $C^{2}$, where $\phi_{0}^{\infty}$ is the corresponding principal eigenfunction.

Note that there is a $V>0$ (independent of $n$ ) and an $n_{2}>n_{0}$ such that $G\left(v ; \varepsilon_{n}\right) \geq\left(\mu_{0}^{\infty}+1\right) v / \lambda_{*}$ for all $v \in[0, V]$ and $n>n_{2}$. If $\phi_{0}^{n}$ is normalised so that $\left\|\phi_{0}^{n}\right\|=V$, then

(10) $-L_{n} \phi_{0}^{n}+\lambda_{n} G\left(\phi_{0}^{n} ; \varepsilon_{n}\right) \geq-L_{n} \phi_{0}^{n}+\lambda_{n}\left(\mu_{0}^{\infty}+1\right) \phi_{0}^{n} / \lambda_{*} \geq\left(\mu_{0}^{\infty}+1-\mu_{0}^{n}\right) \phi_{0}^{n} \geq 0$,

for all $n>n_{2}$ and so $\phi_{0}^{n}$ is a positive subsolution of (9) for all such $n$. An identical argument to the $j=0$ case then leads to a contradiction as before.

We can now prove the following existence result for (3).

Theorem 3.3. Let $\lambda>0$ and $j \in \mathbb{N}_{0}$ be given. Then there exist $\left( \pm u_{j}, \lambda\right) \in \Sigma_{j}^{ \pm}$; that is, $\Pi\left(\Sigma_{j}^{ \pm}\right)=(0, \infty)$.

Proof. Let $\varepsilon_{n} \rightarrow 0$ be any positive sequence. From Lemma 2.2 and Proposition 3.2 with $\lambda_{n} \equiv \lambda$, there is a sequence $u_{n}$ of $C^{2}$ solutions of (4) which is $C^{0}$-bounded and bounded away from zero in $C^{0}$. Since $K u_{n}$ is therefore $C^{2}$-bounded we may pass to a subsequence if necessary and assume that there is a $z \in C^{1}$ such that $K u_{n} \rightarrow z$ in $C^{1}$. Hence, it follows that $\varepsilon_{n} u_{n}+g\left(u_{n}\right) \rightarrow \lambda z$ in $C^{1}$, from where $\varepsilon_{n} u_{n} \rightarrow 0$ in $C^{0}$, so that $g\left(u_{n}\right) \rightarrow \lambda z$ in $C^{0}$. Consequently, $u_{n} \rightarrow g^{-1}(\lambda z)=: u$ in $C^{0}$. Therefore,

$$
\begin{aligned}
\|g(u)-\lambda K u\| & =\left\|\left(g(u)-g\left(u_{n}\right)\right)+\left(g\left(u_{n}\right)-\lambda K u_{n}\right)+\left(\lambda K u_{n}-\lambda K u\right)\right\| \\
& \leq\left\|g(u)-g\left(u_{n}\right)\right\|+\varepsilon_{n}\left\|u_{n}\right\|+\lambda\|K\|\left\|u_{n}-u\right\| \rightarrow 0 .
\end{aligned}
$$


Hence $u$ is a solution of (3). Since $z$ is a $C^{1}$-limit of functions with exactly $j$ transverse zeros we have $\zeta(z)=j$, whence $\zeta(u)=\zeta(g(u))=\zeta(\lambda z)=j$.

3.2. Sequential and continuum bifurcations. We may now establish the existence of an unbounded interval of sequential bifurcation points.

Theorem 3.4. For each $\lambda>0$ there exists a sequence $\left(u_{j}, \lambda\right) \in \Sigma$ such that $\zeta\left(u_{j}\right)=j$ and $u_{j} \rightarrow 0$ in $C^{0}$ as $j \rightarrow \infty$. In particular, every $\lambda \geq 0$ is a sequential bifurcation point for (3).

Proof. Clearly, for each fixed $\lambda>0$ there are infinitely many solutions of (3), $u_{j}$, parameterised by the number of zeros $j \in \mathbb{N}_{0}$. Recall that the corresponding zeros of $g\left(u_{j}\right)$ are transverse. We claim that $\lim _{j \rightarrow \infty} u_{j}=0$ in $C^{0}$. Using the bound $\left\|u_{j}\right\| \leq M(\lambda)$ from Lemma 2.2 we may assume (on passing to a subsequence) that there is a $z \in C^{1}$ such that $K u_{j} \rightarrow z$ in $C^{1}$, so that $g\left(u_{j}\right) \rightarrow \lambda z$ in $C^{1}$ and therefore $u_{j} \rightarrow g^{-1}(\lambda z)$ in $C^{0}$. If $u:=g^{-1}(\lambda z)$, then $u$ is a solution of (3). Since $\zeta\left(g\left(u_{j}\right)\right)=j$, $g(u)$ cannot have finitely many zeros in $\Omega$. Hence by Theorem $2.4 g(u)=0$, from where $z=0$. Hence $g\left(u_{j}\right) \rightarrow 0$ in $C^{1}$ and therefore $u_{j} \rightarrow 0$ in $C^{0}$.

In turn, this implies that $\lambda=0$ is a sequential bifurcation point, simply by setting $\lambda_{n}=1 / n$ and choosing any $\left(\bar{u}_{n}, \lambda_{n}\right) \in \Sigma$ with $\left\|\bar{u}_{n}\right\| \leq 1 / n$.

Next we examine the question of which $\lambda \geq 0$ are continuum bifurcation points.

Lemma 3.5. If $\mathcal{C} \subset \Sigma$ is connected and $(u, \lambda),\left(u^{\prime}, \lambda^{\prime}\right) \in \mathcal{C}$, then $\zeta(u)=\zeta\left(u^{\prime}\right)$.

Proof. Let $(u, \lambda) \in \mathcal{C}$ and suppose that $\left(u_{n}, \lambda_{n}\right) \in \mathcal{C}$ satisfies $\left(u_{n}, \lambda_{n}\right) \rightarrow(u, \lambda)$ as $n \rightarrow \infty$. Using $g\left(u_{n}\right) \equiv \lambda K u_{n}$ we find that $g\left(u_{n}\right) \rightarrow g(u)$ in $C^{1}$ and because $g(u)$ has finitely many transverse zeros, $\zeta\left(u_{n}\right) \zeta\left(g\left(u_{n}\right)\right)=\zeta(g(u))=\zeta(u)$ for all $n$ sufficiently large. This shows that $\zeta(\cdot)$ is an integer-valued continuous function on $\mathcal{C}$ and is therefore constant on $\mathcal{C}$.

Corollary 3.6. For all $\lambda>0, \lambda$ is not a continuum bifurcation point.

Proof. If $\lambda>0$ is a continuum bifurcation point, then there exists a connected set $\mathcal{C} \subset \Sigma$ and a sequence $\left(u_{n}, \lambda_{n}\right) \in \mathcal{C}$ such that $\left(u_{n}, \lambda_{n}\right) \rightarrow(0, \lambda)$ in $E$. By Lemma 3.5 there exists a $j \in \mathbb{N}_{0}$ such that $\left(u_{n}, \lambda_{n}\right) \in \Sigma_{j}$ for all $n$. Passing to a subsequence if necessary, we may assume without loss of generality that $\left(u_{n}, \lambda_{n}\right) \in \Sigma_{j}^{+}$for all $n$. By Proposition 3.2 with $\varepsilon_{n} \equiv 0$ it follows that $\lambda=0$, a contradiction.

Theorem 3.7. $\lambda=0$ is a continuum bifurcation point for (3).

Proof. For each $\lambda>0$ there is a unique $\left(u^{+}, \lambda\right) \in \Sigma_{0}^{+}$by Theorem 3.3 and Lemma 3.1. We prove that the map $\lambda \mapsto u^{+}(\lambda)$ (with $u^{+}(0)=0$ ) from $[0, \infty) \rightarrow C^{0}$ is continuous.

Fix $\lambda \geq 0$ and let $\lambda_{n}>0$ be any sequence satisfying $\lambda_{n} \rightarrow \lambda$ as $n \rightarrow \infty$. Let $u_{n}^{+}:=u^{+}\left(\lambda_{n}\right)$. Suppose that $u^{+}(\cdot)$ is not continuous at $\lambda$; then there is a $\delta>0$ such that $\left\|u_{n}^{+}-u^{+}(\lambda)\right\| \geq \delta$ for all $n$. By Lemma 2.2. $u_{n}^{+}$is bounded in $C^{0}$. From $u_{n}^{+}=\lambda_{n} K g^{-1}\left(u_{n}^{+}\right)$and the compactness of $K$, there exists a convergent subsequence, say $u_{n_{j}}^{+} \rightarrow u^{*}$ in $C^{0}$. Hence $u^{*}$ is a solution of $L u^{*}=\lambda g^{-1}\left(u^{*}\right)$. By Proposition 3.2, if $\lambda>0$, then $u^{*}=u^{+}(\lambda)$, while if $\lambda=0$, then $u^{*}=0$. Either way this contradicts the above $\delta$-bound.

We now utilise a theorem from topological analysis to obtain connectedness results for the sets of non-trivial sign-changing solutions. 
Definition 3.8. Suppose that $(Z, d)$ is a complete metric space and that $\left\{S_{n}\right\}_{n=0}^{\infty}$ is a family of connected subsets of $Z$. For $S \subset Z$ define $d(z, S):=\inf _{s \in S} d(s, z)$,

$$
\begin{aligned}
S_{\mathrm{inf}} & :=\left\{z \in Z: \lim _{n \rightarrow \infty} d\left(z, S_{n}\right)=0\right\}, \\
S_{\mathrm{sup}} & :=\left\{z \in Z: \liminf _{n \rightarrow \infty} d\left(z, S_{n}\right)=0\right\} .
\end{aligned}
$$

Theorem 3.9 (see [17]). Suppose that $\bigcup_{n=0}^{\infty} S_{n}$ is relatively compact in $Z$. If $S_{\mathrm{inf}} \neq \emptyset$, then $S_{\mathrm{sup}}$ is a non-empty, closed and connected subset of $Z$.

Theorem 3.10. Let $j \in \mathbb{N}_{0}$ be given. There exist unbounded, closed and connected sets $\mathcal{C}_{j}^{ \pm} \subset \Sigma_{j}^{ \pm} \cup\{(0,0)\}$ such that $(0,0) \in \mathcal{C}_{j}^{ \pm}$. In particular, $\Pi\left(\mathcal{C}_{j}^{ \pm}\right)=[0, \infty)$.

Proof. Let $\varepsilon_{n} \rightarrow 0$ be any positive sequence. For fixed $\nu>0$ let $S_{n}^{+, j}(\nu)$ be the maximal connected component of $C_{j}^{+}\left(\varepsilon_{n}\right) \cap\left(C^{0} \times[0, \nu]\right)$ which contains $(u, \lambda)=\left(0, \epsilon_{n} \mu_{j}\right)$ in its closure, where $C_{j}(\varepsilon)$ is defined in Theorem 2.3. Note that by Theorem 2.3. $S_{n}^{+, j}(\nu)$ contains non-trivial elements of the form $(u, \lambda)$ for all $\lambda \in\left[\varepsilon_{n} \mu_{j}, \nu\right]$, provided $n$ is sufficiently large and $\left(0, \varepsilon_{n} \mu_{j}\right) \in \overline{S_{n}^{+, j}(\nu)}$. By the compactness of $[0, \nu]$ and of the operator $\left.K: C^{0} \rightarrow C^{0}\right)$ it follows that $\bigcup_{n=0}^{\infty} S_{n}^{+, j}(\nu)$ is relatively compact

in $E$. Clearly $(0,0) \in S_{\text {inf }}^{+, j}(\nu)$ and so $S_{\text {inf }}^{+, j}(\nu)$ is non-empty. Hence by Theorem 3.9 $S_{\text {sup }}^{+, j}(\nu)$ is non-empty, closed and connected in $E$.

Now, by the construction of solutions in Theorem 3.3 it follows that

$$
\left\{\left(u_{j}, \lambda\right) \in \Sigma_{j}^{+}: \lambda \in(0, \nu]\right\} \cup\{(0,0)\} \subset S_{\text {inf }}^{+, j}(\nu) \subset S_{\text {sup }}^{+, j}(\nu) .
$$

Moreover, if $(u, \lambda) \in S_{\text {sup }}^{+, j}(\nu)$ there exists a sequence $\left(u_{n}, \lambda_{n}\right) \in S_{n}^{+, j}(\nu)$ such that $\left(u_{n}, \lambda_{n}\right) \rightarrow(u, \lambda)$ in $E$. Then,

$$
\begin{aligned}
\|g(u)-\lambda K u\| \leq & \left\|g(u)-g\left(u_{n}\right)\right\|+\left|\lambda_{n}-\lambda\right|\left\|K u_{n}\right\| \\
& +\lambda\left\|K\left(u_{n}-u\right)\right\|+\varepsilon_{n}\left\|u_{n}\right\| \rightarrow 0,
\end{aligned}
$$

so that $(u, \lambda)$ is a solution of (3). By Proposition 3.2 and Theorem 2.4 either $(u, \lambda)=(0,0)$ or $(u, \lambda) \in \Sigma_{j}^{+}$for some $j \in \mathbb{N}_{0}$.

Clearly, $S_{\text {sup }}^{+, j}(\nu) \subset S_{\text {sup }}^{+, j}\left(\nu^{\prime}\right)$ if $\nu<\nu^{\prime}$ and it follows that $\mathcal{C}_{j}^{+}: \bigcup_{\nu>0} S_{\text {sup }}^{+, j}(\nu)$ has the stated properties. The result for $\mathcal{C}_{j}^{-}$follows similarly.

Example 1. Consider a semi-linear, degenerate elliptic equation $\Delta \varphi(v)+\lambda f(v)=0$ with Dirichlet boundary conditions on an annulus $R_{1}<|y|<R_{2}$ in $\mathbb{R}^{n}$ [ . Suppose that $\varphi$ and $f$ are strictly increasing, odd functions satisfying $\varphi(0)=f(0)=0$. Setting $u=f(v)$ one obtains $\Delta g(u)+\lambda u=0$, where $g(u):=\varphi\left(f^{-1}(u)\right)$. Suppose that $\varphi$ and $f$ are such that $g$ satisfies G1-G3. Now, radially symmetric solutions satisfy $\left(r^{n-1} g(u)_{r}\right)_{r}+\lambda r^{n-1} u=0$, where $r=|y|$. Setting $x=r^{n} / n$ then yields the equivalent problem $-\left(a(x) g(u)_{x}\right)_{x}=\lambda u$ for $x \in\left(R_{1}^{n} / n, R_{2}^{n} / n\right)$, where $a(x):=$ $(n x)^{2(1-1 / n)}$, to which the results of this section apply. Such a situation occurs when $\varphi(v)=v|v|^{m-1}$ and $f(v)=v|v|^{p-1}$ for $m>p>0$.

\section{Applications}

4.1. Non-monotone eigenvalue problems. Here we apply our main results to problems where $g$ is only locally monotonic near zero. We still obtain infinitely many solution sets in $E$ parameterised by zeros together with an unbounded interval of sequential (but not continuum) bifurcation points. 
Lemma 4.1. Let $\delta>0$ and suppose that $g:[0, \delta] \rightarrow[0, \infty)$ is a strictly increasing $C^{1}$ function which is $C^{2}$ on $(0, \delta]$ with $g(0)=g^{\prime}(0)=0$ and $g^{\prime \prime}(\delta)>0$. If $\gamma(u)=$ $g(u) / u$ satisfies $\gamma^{\prime}(u)>0$ on $(0, \delta]$, then there exists an odd, strictly increasing $C^{1}$ extension $\bar{g}: \mathbb{R} \rightarrow \mathbb{R}$ such that $\left.g\right|_{[0, \delta]}=\left.\bar{g}\right|_{[0, \delta]}$. Moreover, if $\bar{\gamma}(u):=\bar{g}(u) / u$, then $\bar{\gamma}^{\prime}(u)>0$ for all $u>0$ and $\bar{\gamma}(u) \rightarrow \infty$ as $|u| \rightarrow \infty$.

Proof. Since $u^{2} \gamma^{\prime}(u)=u g^{\prime}(u)-g(u)$ we have $g^{\prime}(\delta)>0$. Now define $\bar{g}$ to be the odd extension of the function

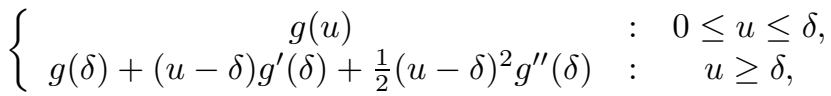

and then for $|u| \geq \delta$ we have $u^{2} \bar{\gamma}^{\prime}(u)=\delta^{2} \gamma^{\prime}(\delta)+\frac{1}{2} g^{\prime \prime}(\delta)\left(u^{2}-\delta^{2}\right)>0$.

We can now deduce the following result when $g$ is only locally monotonic.

Theorem 4.2. For some $\delta>0$ suppose that $g:[-\delta, \delta] \rightarrow \mathbb{R}$ is a strictly increasing, odd, $C^{1}$ function which is $C^{2}$ on $[-\delta, \delta] \backslash\{0\}$ and $g(0)=g^{\prime}(0)=0, g^{\prime \prime}(\delta)>0$. If $\gamma^{\prime}(u) \geq 0$ on $(0, \delta]$, then there exist closed, connected sets $\mathcal{C}_{j}^{ \pm} \subset \Sigma_{j}^{ \pm} \cup\{(0,0)\}$ such that $(0,0) \in \mathcal{C}_{j}^{ \pm}$. At least one, but possibly both, of the following is true:

(1) $\mathcal{C}_{j}^{ \pm}$is unbounded,

(2) there exists $a(u, \lambda) \in \mathcal{C}_{j}^{ \pm}$such that $\|u\|=\delta$.

Furthermore, for each $\lambda>0$ there exists a sequence $u_{j} \in \Sigma$ such that $\zeta\left(u_{j}\right) \rightarrow \infty$ and $u_{j} \rightarrow 0$ in $C^{0}$ as $j \rightarrow \infty$. In particular, every $\lambda \geq 0$ is a sequential bifurcation point and $\lambda=0$ is a continuum bifurcation point for (3).

Proof. Use Lemma 4.1 to replace (3) by $\bar{g}(u)=\lambda K u$ to which Theorems 3.10 and 3.4 apply. The result follows from the fact that solutions of $\bar{g}(u)=\lambda K u$ with $\|u\| \leq \delta$ also satisfy (3).

4.2. Degenerate diffusion equations. Consider a quasi-linear parabolic equations of the form

$$
v_{t}-\left(a(x) D(v)_{x}\right)_{x}+b(x) D(v)=\lambda f(v),
$$

supplied with Dirichlet boundary conditions and given initial data. Such equations arise naturally in many branches of the physical and biological sciences [5, 14. Upon setting $u=f(v)$ and defining $g(u)=D(F(u)$ ) (see below) one may use Theorem 4.2 to obtain information on the existence of equilibrium solutions of (11) whenever $f$ and $D$ are monotonic near zero. We omit the trivial proof.

Theorem 4.3. Suppose that $D, f \in C^{1}(\mathbb{R})$ are odd, strictly increasing functions such that $D(0)=D^{\prime}(0)=f(0)=0$ and $f^{\prime}(0)>0$. Let $F$ denote the local $C^{1}$ inverse of $f$ near 0 . If there exists a $\delta^{*}>0$ such that $D \in C^{2}\left(0, \delta^{*}\right]$ and $u F^{\prime}(u) D^{\prime}(F(u))-$ $D(F(u))>0$ on $\left(0, \delta^{*}\right]$, then the conclusions of Theorem 4.2 hold for equilibrium solutions of (111) for each $\delta \leq \delta^{*}$ for which $(D(F))^{\prime \prime}(\delta)>0$. In particular, the latter conditions hold for all sufficiently small $\delta>0$ whenever $D, f \in C^{3}(\mathbb{R}), D^{\prime \prime}(0)=0$ and $D^{\prime \prime \prime}(0)>0$.

Example 2. Theorem 4.3 applies to a degenerate form of the Chafée-Infante problem (see [6])

$$
v_{t}-\left(v|v|^{m}\right)_{x x}=\lambda v\left(1-v^{2}\right), \quad m>0 .
$$


Example 3. Consider the slow diffusion problem

$$
u_{t}-\left(a(x)[\exp (-1 / u)]_{x}\right)_{x}=\lambda u
$$

with Dirichlet boundary conditions, where $g(u):=[\exp (-1 / u)]$ denotes the odd extension of $\exp (-1 / u)$ for $u>0$. Theorem 4.3 applies to the associated steadystate problem. Note however, that the global results of Section 3 do not apply even though $g$ is globally monotonic due to the failure of the coercivity condition G3. Due to the flat nature of $g$ at $u=0$, the results of [1] do not apply to this equation.

4.3. Boundary value differential-algebraic equations. We can also use the above results to find steady-states of parabolic systems

$$
\begin{aligned}
u_{t}+L u & =\lambda F(u, v), \quad u(0, t)=u(1, t)=0, \\
v_{t} & =G(u, v),
\end{aligned}
$$

or equivalently, the boundary value differential-algebraic equation (DAE)

$$
L u=\lambda F(u, v), G(u, v)=0, \quad u(0)=u(1)=0 .
$$

Problems of this nature are considered in [10], motivated by interactions between diffusive and non-diffusive species. We have the following theorem regarding solutions of (12).

Theorem 4.4. Suppose that $F$ and $G$ are $C^{r}$ functions with $r \geq 4$ such that $F(0,0)=G(0,0)=0, G_{v}(0,0)=G_{v v}(0,0)=0, F(-u,-v)=-F(u, v)$ and $G(u,-v)=-G(-u, v)$. If $G_{u} F_{v} G_{v v v}<0$ at $(0,0)$, then $\lambda=0$ is a continuum bifurcation point to a branch of positive solutions of (12). There are countably many sets of non-trivial solutions $\mathcal{C}_{j} \subset C^{2}(\bar{\Omega}) \times C^{0}(\bar{\Omega}) \times \mathbb{R}$ such that $\mathcal{C}_{j} \cup\{(0,0,0)\}$ is connected, and if $(u, v, \lambda) \in \mathcal{C}_{j}$, then $u$ and $v$ have $j$ zeros in $\Omega$. Every $\lambda \in(0, \infty)$ is a sequential bifurcation point, but no element of $(0, \infty)$ is a continuum bifurcation point.

Proof. Apply the implicit function theorem to $G(u, v)=0$ and solve this constraint as $u=U(v)$, where $U(0)=U^{\prime}(0)=U^{\prime \prime}(0)=0$ and $U^{\prime \prime \prime}(0)=-G_{v v v}(0,0) / G_{u}(0,0)$ $\neq 0$. Then (12) is reduced to $L U(v)=\lambda F(U(v), v)$, so now set $w=F(U(v), v)$. This can be solved by the inverse function theorem for $v=V(w)$ such that $V(0)=0, V^{\prime}(0)=1 / F_{v}(0,0)$ and $V^{\prime \prime}(0)=-F_{v v}(0,0) / F_{v}(0,0)^{3}$. Now, (12) is locally equivalent to $L U(V(w))=\lambda w$, so we set $g(w)=U(V(w))$.

Now, the hypotheses on $F$ and $G$ ensure that $U$ and $V$ are odd functions, so that $g(w)$ is also odd; now set $\gamma(w)=g(w) / w$. Differentiating, we see that $g(w)=$ $\xi w^{3}+o\left(w^{3}\right)$ where $\xi=-G_{v v v} G_{u} F_{v} /\left(G_{u}^{2} F_{v}^{4}\right)>0$ and where each of these derivatives is evaluated at $(u, v)=(0,0)$. Hence there is a $\delta>0$ such that $g(w)>0, \gamma^{\prime}(w)>0$ on $(0, \delta]$ and $g^{\prime \prime}(\delta)>0$. One can now apply Theorem 4.2 to $L g(w)=\lambda w$.

Example 4. The hypotheses of Theorem 4.4 are satisfied by the steady-state problem for the reaction-diffusion system

$$
\begin{aligned}
u_{t}-u_{x x} & =\lambda \sin v, \quad u(0, t)=u(1, t)=0, \\
v_{t} & =u+u^{2} v-v^{3} .
\end{aligned}
$$

Remark 2. Fully non-linear elliptic equations of the form

$$
L u=f(u, L u), \quad u(0)=u(1)=0,
$$

can be written as a boundary value DAE by setting $v=L u, F(u, v)=v$ and $G(u, v)=f(u, v)-v$. Problems of this type are studied, for instance, in [16]. A 
solution of (12) when $\lambda=1$ provides a solution of (13) and these can be obtained using Theorem 4.4 with suitable restrictions on $f$.

\section{REFERENCES}

1. A. Ambrosetti, J. Garcia-Azorero, and I. Peral, Quasilinear equations with a multiple bifurcation, Differential and Integral Equations 10 (1997), no. 1, 37-50. MR 97i:35036]

2. D. Aronson and L.A. Peletier, Large time behaviour of solutions of the porous medium equation in bounded domains, J. Differential Equations 39 (1981), 378-412. MR 82g:35047

3. H. Berestycki, On some nonlinear Sturm-Louiville problems, J. Differential Equations. 26 (1977), 375-390. MR 58:1358

4. H. Berestycki and M.J. Esteban, Existence and bifurcation of solutions for an elliptic degenerate problem, J. Differential Equations 134 (1997), 1-25. MR 97k:34052

5. K.P. Hadeler, Free boundary problems in biology, in Free Boundary Problems: Theory and Applications Vol.II, eds. A. Fasano and M. Primicerio, Pitman Advanced Publishing Program, Pitman, New York, 1983.

6. D. Henry, Geometrical theory of semilinear parabolic equations, Lecture Notes in Mathematics, vol. 840, Springer-Verlag, New York, 1981. MR 83j:35084

7. T. Kato, Perturbation theory for linear operators, vol. Corrected 2nd Edition, Springer-Verlag, New York, 1980. MR 96a:47025

8. M.K. Kwong and L. Zhang, Uniqueness of the positive solution of $\Delta u+f(u)=0$ in an annulus, Differential Integral Equations 4 (1991), 583-596. MR 92b:35015

9. R. Laister and R. E. Beardmore, Transversality and separation of zeros in second order differential equations, Proc. AMS., to appear.

10. M. A. Lewis, Spatial coupling of plant and herbivore dynamics: the contribution of herbivore dispersal to transient and persistant "waves" of damage, Theoretical Population Biology 45 (1994), 277-312.

11. P.L. Lions, Structure of the set of steady-state solutions and asymptotic behaviour of semilinear heat equations, J. Differential Equations 53 (1984), no. 3, 362-386. MR 86b:35092

12. M. Protter and H. Weinberger, Maximum principles in differential equations, Prentice Hall, Englewood Cliffs, N.J. (1967). MR 36:2935

13. P. Rabinowitz, Some global results for nonlinear eigenvalue problems, J. Funct. Anal. 7 (1971), 487-513. MR 46:745

14. A.A. Samarskii, V.A. Galaktionov, S.P. Kurdyumov, and A.P. Mikhailov, Blow-up in quasilinear parabolic equations, de Gruyter Expositions in Mathematics, 19, Walter de Gruyter, Berlin, 1995. MR 96b:35003

15. C.A. Stuart, Bifurcation for Dirichlet problems without eigenvalues, Proc. London Math. Soc. 45 (1982), 169-192. MR 83k:58021

16. S. C. Welsh, A priori bounds and nodal properties for periodic solutions to a class of ordinary differential equations, J. Math. Anal. Appns. 171 (1992), 395-406. MR 93m:34060]

17. G. T. Whyburn, Topological analysis, Princeton University Press, 1964. MR 29:2758

Department of Mathematics, Imperial College, South Kensington, London, SW7 2AZ, UNITED KingDOM

E-mail address: r.beardmore@ic.ac.uk

Department of Mathematics, University of the West of England, Frenchay Campus, Bristol, United Kingdom

E-mail address: robert.laister@uwe.ac.uk 